\title{
ESTIMATES FOR THE GREEN FUNCTION AND EXISTENCE OF POSITIVE SOLUTIONS FOR HIGHER-ORDER ELLIPTIC EQUATIONS
}

IMED BACHAR

Received 7 March 2005; Accepted 7 April 2005

We establish a $3 G$-theorem for the iterated Green function of $(-\Delta)^{p m},(p \geq 1, m \geq 1)$, on the unit ball $B$ of $\mathbb{R}^{n}(n \geq 1)$ with boundary conditions $(\partial / \partial \nu)^{j}(-\Delta)^{k m} u=0$ on $\partial B$, for $0 \leq k \leq p-1$ and $0 \leq j \leq m-1$. We exploit this result to study a class of potentials $\mathscr{J}_{m, n}^{(p)}$. Next, we aim at proving the existence of positive continuous solutions for the following polyharmonic nonlinear problems $(-\Delta)^{p m} u=h(\cdot, u)$, in $D$ (in the sense of distributions), $\lim _{|x| \rightarrow 1}\left((-\Delta)^{k m} u(x) /(1-|x|)^{m-1}\right)=0$, for $0 \leq k \leq p-1$, where $D=B$ or $B \backslash\{0\}$ and $h$ is a Borel measurable function on $D \times(0, \infty)$ satisfying some appropriate conditions related to $\mathscr{E}_{m, n}^{(p)}$.

Copyright (c) 2006 Imed Bachar. This is an open access article distributed under the Creative Commons Attribution License, which permits unrestricted use, distribution, and reproduction in any medium, provided the original work is properly cited.

\section{Introduction}

Let $m \geq 1, p \geq 1$ be a positive integer and $\Gamma_{m, n}^{(p)}$ be the Green function of the polyharmonic operator $u \mapsto(-\triangle)^{p m} u$, on the unit ball $B$ of $\mathbb{R}^{n}(n \geq 1)$ with boundary conditions $(\partial / \partial \nu)^{j}(-\Delta)^{k m} u_{\left.\right|_{\partial B}}=0$, for $0 \leq k \leq p-1$ and $0 \leq j \leq m-1$, where $\partial / \partial v$ is the outward normal derivative.

Then $\Gamma_{m, n}^{(p)}$ satisfies for $p \geq 2$ and each $x, y \in B$,

$$
\Gamma_{m, n}^{(p)}(x, y)=\int_{B} \cdots \int_{B} G_{m, n}\left(x, z_{1}\right) G_{m, n}\left(z_{1}, z_{2}\right) \cdots G_{m, n}\left(z_{p-1}, y\right) d z_{1} \cdots d z_{p-1},
$$

where $G_{m, n}$ is the Green function of the polyharmonic operator $u \mapsto(-\triangle)^{m} u$, on $B$ with Dirichlet boundary conditions $(\partial / \partial \nu)^{j} u=0,0 \leq j \leq m-1$.

In [4], Boggio gave an explicit expression for $G_{m, n}$. In fact, he proved that for each $x, y$ in $B$,

$$
G_{m, n}(x, y)=k_{m, n}|x-y|^{2 m-n} \int_{1}^{[x, y] /|x-y|} \frac{\left(v^{2}-1\right)^{m-1}}{v^{n-1}} d v
$$

Hindawi Publishing Corporation

Abstract and Applied Analysis

Volume 2006, Article ID 89491, Pages 1-22

DOI 10.1155/AAA/2006/89491 
where $k_{m, n}$ is a positive constant and $[x, y]^{2}=|x-y|^{2}+\left(1-|x|^{2}\right)\left(1-|y|^{2}\right)$, for $x, y$ in $B$.

In a recent article, Grunau and Sweers [11] have established interesting estimates for the Green function $\Gamma_{m, n}^{(p)}$. In particular, they proved the existence of a positive constant $C$, such that for each $x, y$ in $B$

$$
\frac{1}{C} H_{m, n}^{(p)}(x, y) \leq \Gamma_{m, n}^{(p)}(x, y) \leq C H_{m, n}^{(p)}(x, y),
$$

where

$$
H_{m, n}^{(p)}(x, y):= \begin{cases}\frac{1}{|x-y|^{n-2 p m} \min \left(1, \frac{(\delta(x) \delta(y))^{m}}{|x-y|^{2 m}}\right)} & \text { if } n>2 p m, \\ \frac{\log \left(1+\frac{(\delta(x) \delta(y))^{m}}{|x-y|^{2 m}}\right)}{\min \left(1,(\delta(x) \delta(y))^{(n-2(p-1) m) / 2} /|x-y|^{n-2(p-1) m}\right)} & \text { if } n=2 p m, \\ (\delta(x) \delta(y))^{(n-2 p m) / 2} & \text { if } 2(p-1) m<n<2 p m, \\ (\delta(x) \delta(y))^{m} \log \left(2+\frac{1}{|x-y|^{2}+\delta(x) \delta(y)}\right) & \text { if } n=2(p-1) m, \\ (\delta(x) \delta(y))^{m} & \text { if } n<2(p-1) m,\end{cases}
$$

and $\delta(x)=1-|x|$, denotes the Euclidean distance between $x$ and $\partial B$.

In the other hand, Grunau and Sweers [10] derived from Boggio's formula some interesting estimates on the Green function $G_{m, n}$ in $B$, including a classical form of a $3 G$ theorem, which holds for the Green function $G_{1, n}^{D}$ of the Laplace operator $u \mapsto(-\Delta) u$, on an arbitrary bounded $C^{1,1}$-domain $D($ see $[6,26])$.

The classical form of the $3 G$-theorem has been exploited to introduce the classical Kato class of functions $K_{n}(D)$, which was widely used in the study of some nonlinear differential equations (see $[18,25]$ ). Definition and properties of the class $K_{n}(D)$ can be found in $[2,6]$.

Recently, in [3] the authors improved the inequalities on $G_{m, n}$ established by Grunau and Sweers in [10]. In particular, they prove the following form of the $3 G$-theorem.

Theorem 1.1 ([3]). There exists $C_{m, n}>0$ such that for each $x, y, z \in B$,

$$
\frac{G_{m, n}(x, z) G_{m, n}(z, y)}{G_{m, n}(x, y)} \leq C_{m, n}\left[\left(\frac{\delta(z)}{\delta(x)}\right)^{m} G_{m, n}(x, z)+\left(\frac{\delta(z)}{\delta(y)}\right)^{m} G_{m, n}(y, z)\right]
$$

This form of the $3 G$-theorem has been proved for the Green function $G_{1, n}^{D}$ in an arbitrary bounded $C^{1,1}$-domain $D$, by Kalton and Verbitsky [12] for $n \geq 3$ and by Selmi [22] for $n=2$. 
In [3], the authors used this inequality (1.5) to introduce and study a new Kato class $K_{m, n}$ of functions on $B$ defined as follows.

Definition 1.2. A Borel measurable function $\varphi$ in $B$ belongs to the class $K_{m, n}$ if $\varphi$ satisfies the following condition

$$
\lim _{\alpha \rightarrow 0}\left(\sup _{x \in B} \int_{B \cap B(x, \alpha)}\left(\frac{\delta(y)}{\delta(x)}\right)^{m} G_{m, n}(x, y)|\varphi(y)| d y\right)=0 .
$$

In the case $m=1$, this class was introduced for a bounded $C^{1,1}$-domain $D$ in $\mathbb{R}^{n}$, in [19] for $n \geq 3$ and in $[15,24]$ for $n=2$. Moreover, it is shown that $K_{1, n}(D)$ contains properly the classical Kato class $K_{n}(D)$.

The plan for this paper is as follows. In Section 2, we give some estimates for the Green function $\Gamma_{m, n}^{(p)}$, which are parallel to those satisfied by the Green function $G_{m, n}$ in $B$ (see $[3,10])$.

In particular, we prove that the Green function $\Gamma_{m, n}^{(p)}$ satisfies the inequality (1.5). This enables us to define and study in Section 3 a Polyharmonic Kato class $\mathscr{E}_{m, n}^{(p)}$ of functions on $B$ (see Definition 1.3 below).

In particular, we prove that $K_{m, n}=\mathscr{E}_{m, n}^{(1)} \subset \mathscr{E}_{m, n}^{(2)} \subset \cdots \subset \mathscr{E}_{m, n}^{(p-1)} \subset \mathscr{F}_{m, n}^{(p)}$ and $\mathscr{E}_{1, n}^{(p)} \subset \cdots \subset$ $\mathscr{F}_{m-1, n}^{(p)} \subset \mathscr{F}_{m, n}^{(p)}$. So we extend some results obtained in [3].

Definition 1.3. A Borel measurable function $\varphi$ in $B$ belongs to the class $\mathscr{E}_{m, n}^{(p)}$ if $\varphi$ satisfies the following condition

$$
\lim _{\alpha \rightarrow 0}\left(\sup _{x \in B} \int_{B \cap B(x, \alpha)}\left(\frac{\delta(y)}{\delta(x)}\right)^{m} \Gamma_{m, n}^{(p)}(x, y)|\varphi(y)| d y\right)=0 .
$$

In Section 4, first we will investigate the existence of positive bounded continuous solutions for the following iterated polyharmonic nonlinear problem

$$
\begin{gathered}
(-\Delta)^{p m} u=f(\cdot, u), \quad \text { in } B \text { (in the sense of distributions) } \\
\lim _{|x| \rightarrow 1} \frac{(-\Delta)^{k m} u(x)}{(1-|x|)^{m-1}}=0, \quad \text { for } 0 \leq k \leq p-1 .
\end{gathered}
$$

We will fix some $r>n$ and we suppose that the function $f$ satisfies the following hypotheses:

$\left(\mathrm{H}_{1}\right) f$ is a nonnegative Borel measurable function on $B \times(0, \infty)$, continuous and nonincreasing with respect to the second variable.

$\left(\mathrm{H}_{2}\right)$ For each $c>0$, the function $x \mapsto f\left(x, c(\delta(x))^{m}\right) /(\delta(x))^{m}$ is in $K_{m, n}$.

$\left(\mathrm{H}_{3}\right)$ For each $c>0$, the function $x \mapsto f\left(x, c(\delta(x))^{m}\right)$ is in $L^{r}(B)$.

$\left(\mathrm{H}_{4}\right)$ For each $c>0, f(\cdot, c)$ is a nontrivial function.

To study problem (1.8), we assume further that $2 \leq n \leq(2 p-1) m$, then we prove that (1.8) has at least one positive solution $u \in C^{2 p m-1}(\bar{B})$ satisfying for all $k \in\{0, \ldots, p-1\}$ and $x \in B$

$$
\frac{1}{c}(\delta(x))^{m} \leq(-\Delta)^{k m} u(x) \leq c(\delta(x))^{m}, \quad \text { for some constant } c>0 .
$$


Recently, Mâagli et al. [17] considered the problem (1.8) when $p=1, m \geq n \geq 2$, and where the function $f$ satisfies similar hypotheses stated above. Then they proved that (1.8) has a positive continuous solution $u$ satisfying $(1 / c)(\delta(x))^{m} \leq u(x) \leq c(\delta(x))^{m-1}$, for some constant $c>0$.

Note that for $m=p=1$, using the complete maximum principle argument, which does not hold for $m \geq 2$, Mâagli and Zribi [18] established an existence and an uniqueness result for the problem (1.8) in a bounded $C^{1,1}$ domain $D$ of $\mathbb{R}^{n}(n \geq 3)$, where the function $f$ is required to satisfy the hypotheses $\left(\mathrm{H}_{1}\right),\left(\mathrm{H}_{4}\right)$ and

$\left(\mathrm{H}_{0}\right)$ For each $c>0, f(\cdot, c)$ is in $K_{n}(D)$.

Here we prove an existence result of the more general problem (1.8) and we obtain estimates both on the solution $u$ and their derivatives $(-\Delta)^{k m} u$, for all $k \in\{1, \ldots, p-1\}$.

As second application we are concerned with the existence of infinitely many singular positive solutions for the following iterated polyharmonic problem

$$
\begin{gathered}
(-\Delta)^{p m} u=\psi(\cdot, u), \quad \text { in } B \backslash\{0\} \text { (in the sense of distributions) } \\
\qquad \lim _{|x| \rightarrow 1} \frac{(-\Delta)^{k m} u(x)}{(1-|x|)^{m-1}}=0, \quad \text { for } 0 \leq k \leq p-1 .
\end{gathered}
$$

We will fix some $r>n$ and we assume the following hypotheses:

$\left(A_{1}\right) \psi$ is a Borel measurable function on $B \times(0, \infty)$, continuous with respect to the second variable.

$\left(\mathrm{A}_{2}\right)|\psi(x, t)| \leq t q(x, t)$, where $q$ is a nonnegative Borel measurable function in $B \times$ $(0, \infty)$ such that the function $t \mapsto q(x, t)$ is nondecreasing on $(0, \infty)$ and $\lim _{t \rightarrow 0} q(x$, $t)=0$.

$\left(\mathrm{A}_{3}\right)$ For each $c>0$, the function $g_{c}$ defined on $B$ by $g_{c}(x)=q\left(x, c G_{m, n}(x, 0)\right)$ belongs to the class $K_{m, n}$.

$\left(\mathrm{A}_{4}\right)$ For each $c>0$, the function $x \mapsto G_{m, n}(x, 0) g_{c}(x)$ is in $L^{r}(B)$.

Then we will prove the existence of $b_{0}>0$ such that for each $b \in\left(0, b_{0}\right]$, the problem (1.10) has a solution $u \in C^{2 p m-1}(\bar{B} \backslash\{0\})$ satisfying for all $k \in\{0, \ldots, p-1\}$ and $x \in B \backslash\{0\}$

$$
\frac{b}{2} \Gamma_{m, n}^{(p-k)}(x, 0) \leq(-\Delta)^{k m} u(x) \leq \frac{3 b}{2} \Gamma_{m, n}^{(p-k)}(x, 0) .
$$

Moreover, for $n \geq 2(p-k) m$, we have

$$
\lim _{|x| \rightarrow 0} \frac{(-\Delta)^{k m} u(x)}{\Gamma_{m, n}^{(p-k)}(x, 0)}=b .
$$

When $m=p=1$ and $D$ is a bounded $C^{1,1}$-domain in $\mathbb{R}^{n}(n \geq 3)$ containing 0 , the existence of infinitely many singular positive solutions for the problem (1.10) has been established by Zhang and Zhao [25] for the special nonlinearity

$$
\psi(x, t)=p(x) t^{\mu}, \quad \mu>1
$$

where the function $p$ satisfies 
$\left(\mathrm{H}_{0}^{\prime}\right)$

$$
x \rightarrow \frac{p(x)}{|x|^{(n-2)(\mu-1)}} \in K_{n}(D) .
$$

This has been extended by Mâagli and Zribi [19], where $\psi$ satisfies some appropriate conditions related to the class $K_{1, n}(D)$.

The case $p=1$ and $m \geq 1$, has been studied in [3].

Here, we extend the result to the more general problem (1.10).

Finally, we aim at proving the existence of positive bounded continuous solutions for the following iterated polyharmonic nonlinear Dirichlet problem

$$
\begin{gathered}
(-\Delta)^{p m} u=\Phi(\cdot, u), \quad \text { in } B \text { (in the sense of distributions) } \\
\lim _{|x| \rightarrow 1} \frac{(-\Delta)^{k m} u(x)}{(1-|x|)^{m-1}}=0, \quad 0 \leq k \leq p-1,
\end{gathered}
$$

where for a given $r>n$, the function $\Phi$ is required to satisfy the following hypotheses:

$\left(\mathrm{R}_{1}\right) \Phi$ is a nonnegative Borel measurable function on $B \times[0, \infty)$, continuous with respect to the second variable.

$\left(\mathrm{R}_{2}\right)$ There exist a nontrivial nonnegative function $h \in L_{\mathrm{loc}}^{1}(B)$ and a nonnegative function $\varphi \in K_{m, n}$ such that

$$
h(x) f(t) \leq \Phi(x, t) \leq(\delta(x))^{m} \varphi(x) g(t),
$$

where $f$ and $g:[0, \infty) \rightarrow[0, \infty)$ are two measurable nondecreasing functions such that

$\left(\mathrm{R}_{2.1}\right)$

$$
\lim _{t \rightarrow 0} \frac{f(t)}{t}=\infty
$$

$\left(\mathrm{R}_{2.2}\right)$

$$
\lim _{t \rightarrow \infty} \frac{g(t)}{t}=0
$$

$\left(\mathrm{R}_{3}\right)$ The function $x \mapsto(\delta(x))^{m} \varphi(x)$ is in $L^{r}(B)$.

Under these hypotheses, we will prove that the problem (1.15) has at least one positive solution $u \in C^{2 p m-1}(\bar{B})$ satisfying for all $k \in\{0, \ldots, p-1\}$ and $x \in B$

$$
\frac{1}{c}(\delta(x))^{m} \leq(-\Delta)^{k m} u(x) \leq c(\delta(x))^{m}, \quad \text { for some constant } c>0 .
$$

Note that the existence of a positive solution of the problem (1.15) in the case $m=1$, $p=2$, has been proved by Peletier and Van der Vorst in [21] for $\Phi(\cdot, t)=|t|^{s}, s>1$ and by Dalmasso in [7] for $\Phi(\cdot, t)=h(t)$, where $h$ is a positive, nondecreasing continuous function on $[0, \infty)$ and sublinear at 0 and $\infty$.

On the other hand, recently Dalmasso obtained in [8] an existence result for the problem (1.15) with $m=1, p=2$, provided that $\Phi$ is locally Hölder continuous function on 
$\bar{B} \times[0, \infty)$ and such that for each $x \in \bar{B}$ the function $\Phi(x, \cdot)$ is nonnegative and nondecreasing on $[0, \infty)$ and $\min _{x \in \bar{B}} \Phi(x, \cdot)$ is sublinear at 0 and $\max _{x \in \bar{B}} \Phi(x, \cdot)$ is sublinear at $\infty$.

In order to simplify our statements, we define some convenient notations.

Notations 1.4. (i) Let $B=\left\{x \in \mathbb{R}^{n} ;|x|<1\right\}$ and $\bar{B}=\left\{x \in \mathbb{R}^{n} ;|x| \leq 1\right\}$, for $n \geq 1$.

(ii) $C(\bar{B})$ is the set of continuous functions in $\bar{B}$.

(iii) $C_{0}(B)$ is the set of continuous functions in $\bar{B}$ vanishing at $\partial B$.

(iv) $C^{k}(B)$ is the set of functions having all derivatives of order $\leq k$ continuous in $B$ $(k \in \mathbb{N})$.

(v) For $x, y \in B$ :

$$
[x, y]^{2}=|x-y|^{2}+\left(1-|x|^{2}\right)\left(1-|y|^{2}\right) \text {. }
$$

(vi) Let $f$ and $g$ be two positive functions on a set $S$.

We call $f \preceq g$, if there is $c>0$ such that

$$
f(x) \leq c g(x), \quad \forall x \in S .
$$

We call $f \sim g$, if there is $c>0$ such that

$$
\frac{1}{c} g(x) \leq f(x) \leq c g(x), \quad \forall x \in S
$$

The following properties will be used several times

(i) For $s, t \geq 0$, we have

$$
\begin{gathered}
\min (s, t) \sim \frac{s t}{s+t}, \\
(s+t)^{\lambda} \sim s^{\lambda}+t^{\lambda}, \quad \lambda \in \mathbb{R}^{+} .
\end{gathered}
$$

(ii) Let $\lambda, \mu>0$ and $0<\gamma \leq 1$, then we have,

$$
\begin{gathered}
1-t^{\lambda} \sim 1-t^{\mu}, \quad \text { for } t \in[0,1] . \\
\log (1+t) \preceq t^{\gamma}, \quad \text { for } t \geq 0 . \\
\log (1+\lambda t) \sim \log (1+\mu t), \quad \text { for } t \geq 0 . \\
\log \left(1+t^{\lambda}\right) \sim \min \left(1, t^{\lambda}\right) \log (2+t), \quad \text { for } t \geq 0 .
\end{gathered}
$$

(iii) On $B^{2}$ (i.e., $\left.(x, y) \in B^{2}\right)$, we have

$$
\begin{gathered}
{[x, y]^{2} \sim|x-y|^{2}+\delta(x) \delta(y)} \\
\delta(x)+\delta(y) \preceq[x, y], \quad|x-y| \leq[x, y] .
\end{gathered}
$$

\section{Estimates for the iterated Green function}

We start this section by proving some inequalities for the iterated Green function $\Gamma_{m, n}^{(p)}$, that we will use later. 
Proposition 2.1. On $B^{2}$, the following estimates hold

$$
\Gamma_{m, n}^{(p)}(x, y) \sim \begin{cases}\frac{(\delta(x) \delta(y))^{m}}{|x-y|^{n-2 p^{m}}[x, y]^{2 m}}, & \text { for } n>2 p m, \\ \frac{(\delta(x) \delta(y))^{m}}{[x, y]^{2 m}} \log \left(1+\frac{[x, y]^{2}}{|x-y|^{2}}\right), & \text { for } n=2 p m \\ \frac{(\delta(x) \delta(y))^{m}}{[x, y]^{n-2(p-1) m},} & \text { for } 2(p-1) m<n<2 p m .\end{cases}
$$

Proof. The proof follows immediately from (1.3) and the statements (1.23), (1.24), (1.29), (1.27), and (1.28).

Using Proposition 2.1, (1.3) and similar argument as in [3, Corollary 2.5] we obtain the following corollary.

Corollary 2.2. Let $r_{0}>0$. For each $x, y \in B$ such that $|x-y| \geq r_{0}$,

$$
\Gamma_{m, n}^{(p)}(x, y) \preceq \frac{(\delta(x) \delta(y))^{m}}{r_{0}{ }^{n-2(p-1) m}} .
$$

Moreover, On $B^{2}$, the following estimates hold:

$$
\begin{gathered}
(\delta(x) \delta(y))^{m} \preceq \Gamma_{m, n}^{(p)}(x, y), \\
\Gamma_{m, n}^{(p)}(x, y) \preceq \frac{\min \left((\delta(x))^{m},(\delta(y))^{m}\right)}{|x-y|^{n-(2 p-1) m}}, \quad \text { if } n>(2 p-1) m, \\
\Gamma_{m, n}^{(p)}(x, y) \preceq \min \left((\delta(x))^{m},(\delta(y))^{m}\right), \quad \text { if } n \leq(2 p-1) m .
\end{gathered}
$$

Corollary 2.3. On $B^{2}$, the following estimates hold:

$$
\left(\frac{\delta(y)}{\delta(x)}\right)^{m} \Gamma_{m, n}^{(p)}(x, y) \preceq \begin{cases}\frac{1}{|x-y|^{n-2 p m}} & \text { for } n>2 p m, \\ \log \left(\frac{3}{|x-y|}\right) & \text { for } n=2 p m, \\ \frac{(\delta(y))^{2 m}}{|x-y|^{n-2(p-1) m}} & \text { for } 2(p-1) m<n<2 p m, \\ (\delta(y))^{2 m-2} & \text { for } n=2(p-1) m, \\ (\delta(y))^{2 m} & \text { for } n<2(p-1) m .\end{cases}
$$

Proof. Using Proposition 2.1 and inequalities (1.30), it is easy to check the statement. 
8 Positive solutions for higher-order equations

Next, we aim at proving inequality $(1.5)$ for the iterated Green function $\Gamma_{m, n}^{(p)}$. So, we need the following key lemma, which is due to [16]. Since [16] is not available we refer to [14] for the proof.

Lemma 2.4. Let $x, y \in B$. Then the following properties are satisfied:

(1) If $\delta(x) \delta(y) \leq|x-y|^{2}$ then $\max (\delta(x), \delta(y)) \leq((\sqrt{5}+1) / 2)|x-y|$.

(2) If $|x-y|^{2} \leq \delta(x) \delta(y)$ then $((3-\sqrt{5}) / 2) \delta(x) \leq \delta(y) \leq((3+\sqrt{5}) / 2) \delta(x)$.

Theorem 2.5 (3G-theorem). There exists a constant $C_{m, n}>0$ such that for each $x, y, z \in B$,

$$
\frac{\Gamma_{m, n}^{(p)}(x, z) \Gamma_{m, n}^{(p)}(z, y)}{\Gamma_{m, n}^{(p)}(x, y)} \leq C_{m, n}\left[\left(\frac{\delta(z)}{\delta(x)}\right)^{m} \Gamma_{m, n}^{(p)}(x, z)+\left(\frac{\delta(z)}{\delta(y)}\right)^{m} \Gamma_{m, n}^{(p)}(y, z)\right]
$$

Proof. To prove the inequality, we denote by $A(x, y):=(\delta(x) \delta(y))^{m} / \Gamma_{m, n}^{(p)}(x, y)$ and we claim that $A$ is a quasi-metric, that is for each $x, y, z \in B$,

$$
A(x, y) \preceq A(x, z)+A(y, z)
$$

To show the claim, we remark that by using (1.3) and Lemma 2.4, we can reproduce the proof of (1.5) in [3, Theorem 2.8]. This completes the proof.

\section{The Polyharmonic Kato class $\mathscr{E}_{m, n}^{(p)}$}

In this section, we will study properties of functions belonging to $\mathscr{F}_{m, n}^{(p)}$.

Example 3.1. For $n>2 p m$ and $s>n / 2 p m, L^{s}(B) \subset \mathscr{E}_{m, n}^{(p)}$.

For $2(p-1) m<n<2 p m$ and $s>n / 2(p-1) m, L^{s}(B) \subset \mathscr{F}_{m, n}^{(p)}$.

For $n \leq 2(p-1) m$ or $n=2 p m, L^{s}(B) \subset \mathscr{g}_{m, n}^{(p)}$, for all $s \in(1, \infty]$.

Using (1.7), (2.3) and similar techniques to those used in [3, Lemma 3.3], we obtain the following lemma.

Lemma 3.2. Let $\varphi$ be a function in $\mathscr{F}_{m, n}^{(p)}$. Then the function

$$
x \longmapsto(\delta(x))^{2 m} \varphi(x) \quad \text { is in } L^{1}(B)
$$

In the sequel, we use the notation

$$
\|\varphi\|_{m, n, p}:=\sup _{x \in B} \int_{B}\left(\frac{\delta(y)}{\delta(x)}\right)^{m} \Gamma_{m, n}^{(p)}(x, y)|\varphi(y)| d y .
$$

Proposition 3.3. Let $\varphi$ be a function in $\mathscr{F}_{m, n}^{(p)}$, then $\|\varphi\|_{m, n, p}<\infty$.

Proof. By writing $B=(B \cap B(x, \alpha)) \cup\left(B \cap B^{c}(x, \alpha)\right)$, then the result follows from (1.7), (2.2), and Lemma 3.2. 
Next we purpose to compare the classes $\mathscr{E}_{j, n}^{(p)},(1 \leq j \leq m)$.

Proposition 3.4. On $B^{2}$, the following estimates hold

$$
\begin{gathered}
\Gamma_{m, n}^{(p)}(x, y) \preceq \Gamma_{m, n}^{(p-1)}(x, y), \quad \text { for } p \geq 2 . \\
\Gamma_{m, n}^{(p)}(x, y) \preceq \delta(x) \delta(y) \Gamma_{m-1, n}^{(p)}(x, y), \quad \text { for } m \geq 2 .
\end{gathered}
$$

In particular,

$$
K_{m, n}=\mathscr{I}_{m, n}^{(1)} \subset \mathscr{F}_{m, n}^{(2)} \cdots \subset \mathscr{F}_{m, n}^{(p)}, \quad \mathscr{E}_{1, n}^{(p)} \subset \mathscr{I}_{2, n}^{(p)} \subset \cdots \subset \mathscr{I}_{m, n}^{(p)} .
$$

Proof. Using (1.1), (1.5) and the fact that $\|\mathbf{1}\|_{m, n, 1}<\infty$, then (3.3) follows by induction on $p$.

The assertion (3.3) implies that $K_{m, n}=\mathscr{J}_{m, n}^{(1)} \subset \mathscr{F}_{m, n}^{(2)} \cdots \subset \mathscr{J}_{m, n}^{(p-1)} \subset \mathscr{F}_{m, n}^{(p)}$.

Next, we will prove (3.4).

Let $x, y \in B$ and $m \geq 2$, then by (1.2), we have

$$
G_{m, n}(x, y) \preceq|x-y|^{2 m-n}\left(\frac{[x, y]^{2}}{|x-y|^{2}}-1\right) \int_{1}^{[x, y] /|x-y|} \frac{\left(v^{2}-1\right)^{m-2} d v}{v^{n-1}} .
$$

Using further (1.29), we deduce that

$$
G_{m, n}(x, y) \preceq \delta(x) \delta(y) G_{m-1, n}(x, y) .
$$

Hence (3.4) follows from this fact and (1.1).

This proves (3.4), which implies that $\mathscr{F}_{j, n}^{(p)} \subset \mathscr{F}_{j+1, n}^{(p)}$, for $j \in\{1, \ldots, m-1\}$.

Remark 3.5. Let $\lambda \in \mathbb{R}$ and put $\theta(x)=1 /(\delta(x))^{\lambda}$, for $x \in B$. Then by [3, Example 3.9], $\theta \in K_{m, n}=\mathscr{I}_{m, n}^{(1)} \Leftrightarrow \lambda<2 m$.

On the other hand, using Lemma 3.2 and [13, lemma, page 726], we deduce that a necessary condition in order that $\theta \in \mathscr{I}_{m, n}^{(p)}$ is $\lambda<2 m+1$.

In fact, for $p \geq 2$, this is sufficient as it will be proved in the following proposition.

Proposition 3.6. Let $p \geq 2$. Then

$$
\theta \in \mathscr{I}_{m, n}^{(p)} \Longleftrightarrow \lambda<2 m+1 .
$$

Proof. “ $\Rightarrow$ ” follows from Remark 3.5.

" $\Leftarrow$ " For $x \in B$ and $\alpha \in(0,1 / 2)$, we put

$$
I=I(x, \alpha):=\int_{B(x, \alpha) \cap B}\left(\frac{\delta(y)}{\delta(x)}\right)^{m} \Gamma_{m, n}^{(p)}(x, y) \frac{1}{(\delta(y))^{\lambda}} d y .
$$

Case 1. Suppose that $\lambda \leq 2 m$.

(a) For $n>2(p-1) m$, using Proposition 2.1, (1.30) and (1.26), it is easy to check that

$$
I \preceq \int_{B(x, \alpha) \cap B} \frac{(\delta(y))^{2 m-\lambda}}{|x-y|^{n-2(p-1) m}} d y .
$$


Hence, since $\lambda \leq 2 m$, it follows that

$$
I \preceq \int_{B(x, \alpha) \cap B} \frac{1}{|x-y|^{n-2(p-1) m}} d y \preceq \alpha^{2(p-1) m} \longrightarrow 0 \quad \text { as } \alpha \longrightarrow 0 .
$$

(b) Let $n=2(p-1) m$, then using (1.3), (1.26) and (1.24), we deduce that

$$
I \preceq \int_{B(x, \alpha) \cap B}\left(1+\frac{1}{[x, y]}\right) d y \preceq \int_{B(x, \alpha) \cap B} \frac{1}{|x-y|} d y \preceq \alpha^{n-1} \longrightarrow 0 \quad \text { as } \alpha \longrightarrow 0 \text {. }
$$

(c) For $n<2(p-1) m$, then by (1.3), we have

$$
I \preceq \int_{B(x, \alpha) \cap B}(\delta(y))^{2 m-\lambda} d y \preceq \alpha^{n} \longrightarrow 0 \quad \text { as } \alpha \longrightarrow 0 .
$$

Case $2(2 m<\lambda<2 m+1)$. (a) If $n>2(p-1) m$, then

$$
\begin{aligned}
I \preceq & \int_{B(x, \alpha) \cap D_{1}} \frac{1}{|x-y|^{n-2(p-1) m}} \frac{1}{(\delta(y))^{\lambda-2 m}} d y \\
& +\int_{B(x, \alpha) \cap D_{2}} \frac{1}{|x-y|^{n-2(p-1) m}} \frac{1}{(\delta(y))^{\lambda-2 m}} d y=I_{1}+I_{2},
\end{aligned}
$$

where $D_{1}=\left\{y \in B:|x-y|^{2} \leq \delta(x) \delta(y)\right\}$ and $D_{2}=\left\{y \in B: \delta(x) \delta(y) \leq|x-y|^{2}\right\}$.

$\left(\mathrm{a}_{1}\right) y \in D_{1}$. Using Lemma 2.4, we have $\delta(x) \sim \delta(y)$ and so $|x-y| \preceq \delta(y)$.

Hence,

$$
I_{1} \preceq \int_{B(x, \alpha) \cap D_{1}} \frac{1}{|x-y|^{n-2 p m+\lambda}} d y \preceq \alpha^{2 p m-\lambda} \longrightarrow 0 \quad \text { as } \alpha \longrightarrow 0
$$

(a $) y \in D_{2}$. Using Lemma 2.4, we have $(1-|y|) \leq((\sqrt{5}+1) / 2)|x-y|$.

Hence,

$$
I_{2} \preceq \int_{1-\alpha((\sqrt{5}+1) / 2)}^{1} \frac{t^{n-1}}{(1-t)^{\lambda-2 m}}\left(\int_{S^{n-1}} \frac{1}{|x-t \omega|^{n-2(p-1) m}} d \sigma(w)\right) d t
$$

where $\sigma$ is the normalized measure on the unit sphere $S^{n-1}$ of $\mathbb{R}^{n}$.

Now it is easy to check that

$$
\int_{S^{n-1}} \frac{1}{|x-t \omega|^{n-2(p-1) m}} d \sigma(w) \preceq \frac{1}{(|x| \vee t)^{n-2(p-1) m}},
$$

where $|x| \vee t=\max (|x|, t)$ 
Hence

$$
I_{2} \preceq \int_{1-\alpha((\sqrt{5}+1) / 2)}^{1} \frac{t^{2(p-1) m-1}}{(1-t)^{\lambda-2 m}} d t \longrightarrow 0 \quad \text { as } \alpha \longrightarrow 0 .
$$

(b) If $n=2(p-1) m$, then using (1.3), we have

$$
\begin{aligned}
I \preceq & \int_{B(x, \alpha) \cap D_{1}} \log \left(2+\frac{1}{|x-y|^{2}+\delta(x) \delta(y)}\right) \frac{1}{(\delta(y))^{\lambda-2 m}} d y \\
& +\int_{B(x, \alpha) \cap D_{2}} \log \left(2+\frac{1}{|x-y|^{2}+\delta(x) \delta(y)}\right) \frac{1}{(\delta(y))^{\lambda-2 m}} d y \\
= & I_{1}+I_{2},
\end{aligned}
$$

where $D_{1}$ and $D_{2}$ are given in Case 2(a).

$\left(\mathrm{b}_{1}\right) y \in D_{1}$. Using Lemma 2.4, we have $\delta(x) \sim \delta(y)$ and so $|x-y| \preceq \delta(y)$.

Hence, by (1.3), (1.26) and (1.24), we deduce that

$$
\begin{aligned}
I_{1} & \preceq \int_{B(x, \alpha) \cap D_{1}} \frac{1}{|x-y|} \frac{1}{(\delta(y))^{\lambda-2 m}} d y \\
& \preceq \int_{B(x, \alpha) \cap D_{1}} \frac{1}{|x-y|^{\lambda-2 m+1}} d y \preceq \alpha^{2 p m-\lambda-1} \longrightarrow 0 \quad \text { as } \alpha \longrightarrow 0 .
\end{aligned}
$$

$\left(\mathrm{b}_{2}\right) y \in D_{2}$. Using Lemma 2.4, we have $\delta(y) \leq((\sqrt{5}+1) / 2)|x-y|$.

Hence,

$$
\begin{aligned}
I_{2} & \preceq \int_{(1-\alpha((\sqrt{5}+1) / 2) \leq|y| \leq 1)} \log \left(2+\frac{3}{(\delta(y))^{2}}\right) \frac{1}{(\delta(y))^{\lambda-2 m}} d y \\
& \preceq \int_{1-\alpha((\sqrt{5}+1) / 2)}^{1} \frac{t^{n-1}}{(1-t)^{\lambda-2 m}} \log \left(2+\frac{3}{(1-t)^{2}}\right) d t \longrightarrow 0 \quad \text { as } \alpha \longrightarrow 0 .
\end{aligned}
$$

(c) If $n<2(p-1) m$, then the result follows by using (1.3), Lemma 2.4 and similar argument to Case $2(\mathrm{a})$.

Corollary 3.7. For $p \geq 2$, the class $\mathscr{F}_{m, n}^{(p)}$ properly contains $K_{m, n}$.

Proof. The assertion follows from (3.5), Remark 3.5 and Proposition 3.6.

\section{Existence results}

We are concerned with the existence of positive continuous solutions for the iterated polyharmonic nonlinear problems (1.8) and (1.10). The following preliminary results will be used in the sequel.

Proposition 4.1. Let $x_{0} \in \bar{B}$ and $\varphi$ be a function in $\mathscr{g}_{m, n}^{(p)}$. Then

$$
\lim _{\alpha \rightarrow 0}\left(\sup _{x \in B} \int_{B\left(x_{0}, \alpha\right) \cap B}\left(\frac{\delta(y)}{\delta(x)}\right)^{m} \Gamma_{m, n}^{(p)}(x, y)|\varphi(y)| d y\right)=0 .
$$


12 Positive solutions for higher-order equations

Proof. Let $\varepsilon>0$, then by (1.7) and (2.2) there exists $\alpha>0$ such that

$$
\int_{B\left(x_{0}, \alpha\right) \cap B}\left(\frac{\delta(y)}{\delta(x)}\right)^{m} \Gamma_{m, n}^{(p)}(x, y)|\varphi(y)| d y \preceq \varepsilon+\int_{B\left(x_{0}, \alpha\right) \cap B \cap B^{c}(x, \alpha)}(\delta(y))^{2 m}|\varphi(y)| d y .
$$

Hence using Lemma 3.2, the result holds by letting $\alpha \rightarrow 0$.

Corollary 4.2. Let $\varphi \in \mathscr{F}_{m, n}^{(p)}$ and $x_{0} \in \bar{B}$. Then

$$
\lim _{\alpha \rightarrow 0}\left(\sup _{x, y \in B} \frac{1}{\Gamma_{m, n}^{(p)}(x, y)} \int_{B \cap B\left(x_{0}, \alpha\right)} \Gamma_{m, n}^{(p)}(x, z) \Gamma_{m, n}^{(p)}(z, y)|\varphi(z)| d z\right)=0 .
$$

Corollary 4.3. Let $\varphi$ be a function in $\mathscr{F}_{m, n}^{(p)}$. Then the function

$$
x \longmapsto \Phi(x):=\int_{B}\left(\frac{\delta(y)}{\delta(x)}\right)^{m} \Gamma_{m, n}^{(p)}(x, y) \varphi(y) d y, \quad \text { is continuous on } \bar{B} .
$$

Proof. Let $x_{0} \in \bar{B}$ and $\varepsilon>0$. By Proposition 4.1, there exists $\alpha>0$ such that for each $x, x^{\prime} \in$ $B\left(x_{0}, \alpha\right) \cap B$, we have

$$
\begin{aligned}
\left|\Phi(x)-\Phi\left(x^{\prime}\right)\right| & \leq \int_{B}\left|\frac{\Gamma_{m, n}^{(p)}(x, y)}{(\delta(x))^{m}}-\frac{\Gamma_{m, n}^{(p)}\left(x^{\prime}, y\right)}{\left(\delta\left(x^{\prime}\right)\right)^{m}}\right|(\delta(y))^{m}|\varphi(y)| d y \\
& \leq \varepsilon+\int_{\left(\left|x_{0}-y\right| \geq 2 \alpha\right) \cap B}\left|\frac{\Gamma_{m, n}^{(p)}(x, y)}{(\delta(x))^{m}}-\frac{\Gamma_{m, n}^{(p)}\left(x^{\prime}, y\right)}{\left(\delta\left(x^{\prime}\right)\right)^{m}}\right|(\delta(y))^{m}|\varphi(y)| d y .
\end{aligned}
$$

Now since for $y \in B^{c}\left(x_{0}, 2 \alpha\right)$, the function $x \mapsto(\delta(y) / \delta(x))^{m} \Gamma_{m, n}^{(p)}(x, y)$ is continuous on $B\left(x_{0}, \alpha\right) \cap B$, we deduce by Lemma 3.2 and the dominated convergence theorem that

$$
\int_{\left(\left|x_{0}-y\right| \geq 2 \alpha\right) \cap B}\left|\frac{\Gamma_{m, n}^{(p)}(x, y)}{(\delta(x))^{m}}-\frac{\Gamma_{m, n}^{(p)}\left(x^{\prime}, y\right)}{\left(\delta\left(x^{\prime}\right)\right)^{m}}\right|(\delta(y))^{m}|\varphi(y)| d y \longrightarrow 0 \quad \text { as }\left|x-x^{\prime}\right| \longrightarrow 0 .
$$

Hence $\Phi \in C(\bar{B})$. This completes the proof.

The next remark will be used to obtain regularity of the solution.

Remark 4.4. Let $r>n$ and $f$ be a nonnegative measurable function in $L^{r}(B)$. Let

$$
V_{m, n} f(x)=\int_{B} \Gamma_{m, n}^{(p)}(x, y) f(y) d y, \quad \text { for } x \in B .
$$

Then $V_{m, n} f \in C^{2 p m-1}(\bar{B})$. 
Indeed, by using the regularity theory of [1] (see also [10, Theorem 5.1] and [5, Theorem IX.32]) we obtain that $V_{m, n} f \in W^{2 p m, r}(B)$. Furthermore, since $r>n$, then one finds that $V_{m, n} f \in C^{2 p m-1}(\bar{B})$ (see [9, Chapter 7, page 158] or [5, Corollary IX.15]).

4.1. Positive bounded solutions of the problem (1.8). We consider the case $2 \leq n \leq$ $(2 p-1) m$ to study the existence of positive bounded continuous solutions for the iterated polyharmonic nonlinear problem (1.8).

We start with the following existence result.

Theorem 4.5. Let $\lambda>0$ and assume $\left(H_{1}\right),\left(H_{2}\right)$ and $\left(H_{4}\right)$. Then the problem

$$
\begin{gathered}
(-\Delta)^{p m} u=f(\cdot, u), \quad \text { in } B(\text { in the sense of distributions }) \\
\lim _{|x| \rightarrow 1} \frac{u(x)-\lambda}{(1-|x|)^{m-1}}=0,
\end{gathered}
$$

has at least one positive solutions $u_{\lambda} \in C(\bar{B})$.

Proof. Let $\lambda>0$. Then by $\left(\mathrm{H}_{1}\right)$ and $\left(\mathrm{H}_{2}\right)$, the function $\varphi(y):=f(y, \lambda) /(\delta(y))^{m} \in K_{m, n}$. So by (3.5) and Proposition 3.3, we have $\|\varphi\|_{m, n, 1}<\infty$. Let $F$ be the convex set given by

$$
F=\left\{u \in C(\bar{B}): \lambda \leq u \leq \lambda+(\delta(x))^{m}\|\varphi\|_{m, n, 1}\right\}
$$

We consider the integral operator $T$ on $F$, defined by

$$
T u(x)=\lambda+\int_{B} \Gamma_{m, n}^{(p)}(x, y) f(y, u(y)) d y
$$

We will prove that $T$ has a fixed point in $F$. Since for $u \in F$ and $y \in B$, we have by $\left(\mathrm{H}_{1}\right)$

$$
\frac{f(y, u(y))}{(\delta(y))^{m}} \leq \frac{f(y, \lambda)}{(\delta(y))^{m}}=\varphi(y),
$$

then by Corollary 4.3 , we deduce that the family $T F$ is equicontinuous in $\bar{B}$. In particular, for all $u \in F, T u \in C(\bar{B})$ and so it is clear that $T F \subset F$. Moreover, the family $\{T u(x), u \in$ $F$ \} is uniformly bounded in $\bar{B}$. It follows by Ascoli's theorem that $T F$ is relatively compact in $C(\bar{B})$.

Next, let us prove the continuity of $T$ in $F$. We consider a sequence $\left\{v_{k}\right\}$ in $F$ which converges uniformly to a function $v \in F$. Then we have

$$
\left|T v_{k}(x)-T v(x)\right| \leq \int_{B} \Gamma_{m, n}^{(p)}(x, y)\left|f\left(y, v_{k}(y)\right)-f(y, v(y))\right| d y .
$$

Now, by the monotonicity of $f$, we have

$$
\left|f\left(y, v_{k}(y)\right)-f(y, v(y))\right| \leq 2 f(y, \lambda)=2(\delta(y))^{m} \varphi(y),
$$


and since $f$ is continuous with respect to the second variable, we deduce by (2.5), Lemma 3.2 and the dominated convergence theorem, that

$$
\forall x \in B, \quad T v_{k}(x) \longrightarrow T v(x) \text { as } k \longrightarrow \infty
$$

Since $T F$ is relatively compact in $C(\bar{B})$, we have the uniform convergence, namely

$$
\left\|T v_{k}-T v\right\|_{\infty} \longrightarrow 0 \quad \text { as } k \rightarrow \infty
$$

Thus we have proved that $T$ is a compact mapping from $F$ to itself. Hence by Schäuder fixed point theorem, there exists $u_{\lambda} \in F$ such that

$$
u_{\lambda}(x)=\lambda+\int_{B} \Gamma_{m, n}^{(p)}(x, y) f\left(y, u_{\lambda}(y)\right) d y
$$

Finally, we need to verify that $u_{\lambda}$ is a solution for the problem $\left(P_{\lambda}\right)$. Since by $\left(\mathrm{H}_{1}\right)$ we have for each $y \in B, f\left(y, u_{\lambda}(y)\right) \leq f(y, \lambda)=(\delta(y))^{m} \varphi(y)$, then we deduce from Lemma 3.2, that the function $y \mapsto f\left(y, u_{\lambda}(y)\right)$ is in $L_{\text {loc }}^{1}(B)$. So it is clear that $u_{\lambda}$ satisfies (in the sense of distributions) the elliptic differential equation

$$
(-\Delta)^{p m} u=f(\cdot, u) \quad \text { in } B
$$

Moreover, since $u_{\lambda} \in F$, then it satisfies

$$
0 \leq \frac{u_{\lambda}(x)-\lambda}{(1-|x|)^{m-1}} \preceq \delta(x) .
$$

Hence $\lim _{|x| \rightarrow 1}\left(u_{\lambda}(x)-\lambda /(1-|x|)^{m-1}\right)=0$. This completes the proof.

In the sequel, we consider a sequence $\left(\lambda_{k}\right)_{k}$ of positive real numbers, decreasing to zero. We denote by $u_{k}$ the solution of the problem $\left(P_{\lambda_{k}}\right)$ given by Theorem 4.5 and satisfying (4.15). That is

$$
u_{k}(x)=\lambda_{k}+\int_{B} \Gamma_{m, n}^{(p)}(x, y) f\left(y, u_{k}(y)\right) d y, \quad \forall k \in \mathbb{N}, \forall x \in B .
$$

Lemma 4.6. There exists a positive constant a such that for all $k \in \mathbb{N}$, and $x \in B, u_{k}(x) \geq$ $a(\delta(x))^{m}$.

Proof. By (2.3) and (2.5), we remark that for $2 \leq n \leq(2 p-1) m$, we have on $B$

$$
\Gamma_{m, n}^{(p)}(0, y) \sim(\delta(y))^{m}
$$

Then again by (2.3) and (2.5), we deduce that there exists a positive constant $c>1$ such that we have for each $x, y \in B$

$$
\frac{1}{c}(\delta(x))^{m} \Gamma_{m, n}^{(p)}(0, y) \leq \Gamma_{m, n}^{(p)}(x, y) \leq c \Gamma_{m, n}^{(p)}(0, y)
$$


This implies by (4.18) that

$$
\begin{aligned}
u_{k}(x) & \leq c\left(\lambda_{k}+\int_{B} \Gamma_{m, n}^{(p)}(0, y) f\left(y, u_{k}(y)\right) d y\right)=c u_{k}(0), \\
u_{k}(x) & \geq \frac{1}{c}(\delta(x))^{m}\left(\lambda_{k}+\int_{B} \Gamma_{m, n}^{(p)}(0, y) f\left(y, u_{k}(y)\right) d y\right) \\
& \geq \frac{1}{c}(\delta(x))^{m}\left(\inf _{k \in \mathbb{N}} u_{k}(0)\right) .
\end{aligned}
$$

We claim that $a=(1 / c)\left(\inf _{k \in \mathbb{N}} \mathcal{u}_{k}(0)\right)>0$. Assume on the contrary that there exists a subsequence $\left(u_{k_{s}}(0)\right)_{s}$ which converges to zero. In particular, for $s$ large enough, we have $u_{k_{s}}(0) \leq 1$. Which implies with $(4.21)$ and $\left(\mathrm{H}_{1}\right)$ that

$$
u_{k_{s}}(0)=\lambda_{k_{s}}+\int_{B} \Gamma_{m, n}^{(p)}(0, y) f\left(y, u_{k_{s}}(y)\right) d y \geq \lambda_{k_{s}}+\int_{B} \Gamma_{m, n}^{(p)}(0, y) f(y, c) d y .
$$

Thus, by letting $s$ to $\infty$, we reach a contradiction from hypothesis $\left(\mathrm{H}_{4}\right)$. This completes the proof.

Now we are ready to prove the existence result for the problem (1.8).

Theorem 4.7. Assume $\left(H_{1}\right)-\left(H_{4}\right)$. Then the problem (1.8) has at least one positive solution $u \in C^{2 p m-1}(\bar{B})$, satisfying for all $k \in\{0, \ldots, p-1\}$

$$
(-\Delta)^{k m} u(x) \sim(\delta(x))^{m}
$$

Proof. Let $\left(u_{k}\right)_{k}$ the sequence of functions satisfying (4.18) and $a$ be the constant given in Lemma 4.6. By hypothesis $\left(\mathrm{H}_{2}\right)$, we deduce that the function

$$
\varphi(y):=\frac{f\left(y, a(\delta(y))^{m}\right)}{(\delta(y))^{m}} \in K_{m, n} .
$$

Since for each $k \in \mathbb{N}$ and $y \in B$, we have by $\left(\mathrm{H}_{1}\right)$

$$
\frac{f\left(y, u_{k}(y)\right)}{(\delta(y))^{m}} \leq \frac{f\left(y, a(\delta(y))^{m}\right)}{(\delta(y))^{m}}=\varphi(y),
$$

then using (4.18) and similar argument as in the proof of Theorem 4.5, we deduce that the family $\left(u_{k}\right)_{k}$ is relatively compact in $C(\bar{B})$. Then it follows that there exists a subsequence $\left(u_{k_{s}}\right)_{s}$ which converges uniformly to a function $u \in C(\bar{B})$. Moreover, by Lemma 4.6, we have $u(x) \geq a(\delta(x))^{m}$, for each $x \in B$. Hence, using the continuity of $f$ with respect to the second variable, we apply the dominated convergence theorem in (4.18) to obtain that

$$
u(x)=\int_{B} \Gamma_{m, n}^{(p)}(x, y) f(y, u(y)) d y .
$$

Using Lemma 4.6, $\left(\mathrm{H}_{1}\right)$, and $\left(\mathrm{H}_{3}\right)$ we obtain for each $y \in B$,

$$
f(y, u(y)) \leq f\left(y, a(\delta(y))^{m}\right)=(\delta(y))^{m} \varphi(y) \in L^{r}(B) .
$$


So it is clear that $u$ satisfies (in the sense of distributions) the elliptic differential equation

$$
(-\Delta)^{p m} u=f(\cdot, u), \quad \text { in } B
$$

Furthermore, by (4.27), (4.28), and Remark 4.4, we deduce that $u \in C^{2 p m-1}(\bar{B})$.

Therefore, using (4.27) and (1.1) it is easy to check that

$$
(-\Delta)^{k m} u(x)=\int_{B} \Gamma_{m, n}^{(p-k)}(x, y) f(y, u(y)) d y, \quad \forall k \in\{0, \ldots, p-1\}
$$

Now since by $\left(\mathrm{H}_{2}\right)$, the function $\varphi \in K_{m, n}$, then it follows by [3, Proposition 3.4] that $\|\varphi\|_{m, n, 1}<\infty$. So using (4.30), (4.28), and (3.3), there exists $c>0$, such that for all $k \in$ $\{0, \ldots, p-1\}$ and $x \in B$, we have

$$
(-\Delta)^{k m} u(x) \leq c \int_{B} G_{m, n}(x, y)(\delta(y))^{m} \varphi(y) d y \leq c(\delta(x))^{m}\|\varphi\|_{m, n, 1}
$$

On the other hand, from (4.30), (2.3) and $\left(\mathrm{H}_{1}\right)$, there exists $c_{0}>0$, such that for all $k \in$ $\{0, \ldots, p-1\}$ and $x \in B$, we have

$$
(-\Delta)^{k m} u(x) \geq c_{0}(\delta(x))^{m} \int_{B}(\delta(y))^{m} f\left(y, c(\delta(y))^{m}\|\varphi\|_{m, n, 1}\right) d y
$$

Now by $\left(\mathrm{H}_{3}\right)$, we deduce that

$$
\int_{B}(\delta(y))^{m} f\left(y, c(\delta(y))^{m}\|\varphi\|_{m, n, 1}\right) d y<\infty
$$

That is $(-\Delta)^{k m} u(x) \sim(\delta(x))^{m}$ and $u$ is the required solution.

Example 4.8. Assume that $2 \leq n \leq(2 p-1) m$. Let $\alpha>0, r>n$ and $\lambda<1 / r$. Let $\rho$ be a nonnegative nontrivial measurable function in $B$ such that $0 \leq \rho(x) \leq(\delta(x))^{m \alpha-\lambda}$, for $x \in B$. Then the following problem

$$
\begin{gathered}
(-\Delta)^{p m} u=\rho(x) u^{-\alpha}, \quad \text { in } B \text { (in the sense of distributions) } \\
\lim _{|x| \rightarrow 1} \frac{(-\Delta)^{k m} u(x)}{(1-|x|)^{m-1}}=0, \quad \text { for } 0 \leq k \leq p-1
\end{gathered}
$$

has a positive solution $u \in C^{2 p m-1}(\bar{B})$ satisfying for all $k \in\{0, \ldots, p-1\}$

$$
(-\Delta)^{k m} u(x) \sim(\delta(x))^{m} .
$$


4.2. Positive singular solutions of the problem (1.10). We are interested in the existence of positive singular solutions for the iterated polyharmonic nonlinear problem (1.10). Our main result is the following theorem.

Theorem 4.9. Assume $\left(A_{1}\right)-\left(A_{4}\right)$. Then the problem (1.10) has infinitely many solutions. More precisely, there exists $b_{0}>0$ such that for each $b \in\left(0, b_{0}\right]$, the problem (1.10) has a solution $u \in C^{2 p m-1}(\bar{B} \backslash\{0\})$ satisfying for all $k \in\{0, \ldots, p-1\}$ and $x \in B$

$$
\frac{b}{2} \Gamma_{m, n}^{(p-k)}(x, 0) \leq(-\Delta)^{k m} u(x) \leq \frac{3 b}{2} \Gamma_{m, n}^{(p-k)}(x, 0)
$$

Moreover, for $n \geq 2(p-k) m$ we have $\lim _{|x| \rightarrow 0}\left((-\Delta)^{k m} u(x) / \Gamma_{m, n}^{(p-k)}(x, 0)\right)=b$.

To prove Theorem 4.9, we need the following results.

Next, for a nonnegative function $\rho \in K_{m, n}$ we denote by

$$
\mathfrak{M}_{\rho}=\left\{h \in K_{m, n},|h| \leq \rho\right\}
$$

Then we have the following proposition.

Proposition 4.10. Let $k \in\{0,1, \ldots, p-1\}$ and $\rho$ be a nonnegative function in $K_{m, n}$. Then the family of functions

$$
\left\{\int_{B} \frac{\Gamma_{m, n}^{(p-k)}(x, y) \Gamma_{m, n}^{(p-k)}(y, 0)}{\Gamma_{m, n}^{(p-k)}(x, 0)} h(y) d y: h \in \mathfrak{M}_{\rho}\right\}
$$

is uniformly bounded and equicontinuous on $\bar{B}$, and consequently it is relatively compact in $C(\bar{B})$.

Proof. Using Theorem 2.5, (3.3) and (2.2), the proof follows by similar arguments as in [3, Lemma 4.3].

Put $F:=\left\{\omega \in C^{+}(\bar{B}):\|\omega\|_{\infty} \leq 1\right\}$, where $\|\cdot\|_{\infty}$ is the uniform norm. So we obtain the following corollary.

Corollary 4.11. Assume $\left(A_{1}\right)-\left(A_{3}\right)$. For $k \in\{0,1, \ldots, p-1\}$, we define the operator $T_{k}$ on F by

$$
T_{k} \omega(x)=\frac{1}{\Gamma_{m, n}^{(p-k)}(x, 0)} \int_{B} \Gamma_{m, n}^{(p-k)}(x, y) \psi\left(y, \omega(y) \Gamma_{m, n}^{(p)}(y, 0)\right) d y, \quad x \in B .
$$

Then the family of functions $T_{k}(F)$ is relatively compact in $C(\bar{B})$.

Proof. Since by $\left(\mathrm{A}_{2}\right)$ and (3.3) there exists a constant $c>0$ such that for each $k \in\{0, \ldots$, $p-1\},\left|\psi\left(y, \omega(y) \Gamma_{m, n}^{(p)}(y, 0)\right) / \Gamma_{m, n}^{(p-k)}(y, 0)\right| \preceq g_{c}(x)=q\left(x, c G_{m, n}(x, 0)\right)$, then the result follows from $\left(\mathrm{A}_{3}\right)$ and Proposition 4.10. 
Remark 4.12. Let $k \in\{0, \ldots, p-1\}, n \geq 2(p-k) m$ and $\alpha>0$. Then for $y \in B^{c}(0,2 \alpha) \cap B$, we have

$$
\lim _{|x| \rightarrow 0} \frac{\Gamma_{m, n}^{(p-k)}(x, y)}{\Gamma_{m, n}^{(p-k)}(x, 0)}=0
$$

So, using the same argument as in the proof of Proposition 4.10, we deduce that $\left|T_{k} \omega(x)\right|$ $\rightarrow 0$ as $|x| \rightarrow 0$, uniformly for all $\omega \in F$.

Now we are ready to prove Theorem 4.9.

Proof of Theorem 4.9. We aim at proving the existence of a constant $b_{0}>0$ such that for each $b \in\left(0, b_{0}\right]$, there exists a continuous function $u$ in $B \backslash\{0\}$ satisfying the following integral equation

$$
u(x)=b \Gamma_{m, n}^{(p)}(x, 0)+\int_{B} \Gamma_{m, n}^{(p)}(x, y) \psi(y, u(y)) d y, \quad x \in B \backslash\{0\} .
$$

Let $k \in\{0,1, \ldots, p-1\}$ and $\beta \in(0,1)$. Then by Proposition 4.10 , we deduce that the function

$$
T_{k, \beta}(x)=\frac{1}{\Gamma_{m, n}^{(p-k)}(x, 0)} \int_{B} \Gamma_{m, n}^{(p-k)}(x, y) \Gamma_{m, n}^{(p-k)}(y, 0) q\left(y, \beta \Gamma_{m, n}^{(p)}(y, 0)\right) d y
$$

is continuous in $\bar{B}$. Moreover, by $\left(\mathrm{A}_{3}\right)$, Proposition 3.3, $\left(\mathrm{A}_{2}\right)$ and the dominated convergence theorem we deduce that

$$
\lim _{\beta \rightarrow 0} T_{k, \beta}(x)=0, \quad \forall x \in \bar{B}
$$

Since the function $\beta \rightarrow T_{k, \beta}(x)$ is nondecreasing in $(0,1)$, it follows by Dini lemma, that

$$
\lim _{\beta \rightarrow 0}\left(\sup _{x \in B} T_{k, \beta}(x)\right)=0
$$

Using further (3.3) and that the function $t \rightarrow q(x, t)$ is nondecreasing in $(0, \infty)$, there exists $\beta \in(0,1)$ such that for each $l \in\{0, \ldots, p-1\}$ and $x \in \bar{B}$,

$$
\frac{1}{\Gamma_{m, n}^{(p-l)}(x, 0)} \int_{B} \Gamma_{m, n}^{(p-l)}(x, y) \Gamma_{m, n}^{(p)}(y, 0) q\left(y, \beta \Gamma_{m, n}^{(p)}(y, 0)\right) d y \leq \frac{1}{3} .
$$

Let $b_{0}=(2 / 3) \beta$ and $b \in\left(0, b_{0}\right]$. We will use a fixed point argument. Let

$$
S=\left\{\omega \in C(\bar{B}): \frac{b}{2} \leq \omega(x) \leq \frac{3 b}{2}\right\}
$$


Then, $S$ is a nonempty, closed, bounded, and convex set in $C(\bar{B})$. We define for $0 \leq k \leq$ $p-1$, the operator $L_{k}$ on $S$ by

$$
L_{k} \omega(x)=b+\frac{1}{\Gamma_{m, n}^{(p-k)}(x, 0)} \int_{B} \Gamma_{m, n}^{(p-k)}(x, y) \psi\left(y, \omega(y) \Gamma_{m, n}^{(p)}(y, 0)\right) d y, \quad \forall x \in B .
$$

By Corollary 4.11 and $\left(\mathrm{A}_{2}\right)$ it is easy to verify that $L_{k}$ is a compact operator mapping $S$ into itself. So by the Schäuder fixed point theorem there exists of $\omega_{0} \in S$ such that $L_{0} \omega_{0}=\omega_{0}$.

For all $x \in B \backslash\{0\}$, put $u(x)=\omega_{0}(x) \Gamma_{m, n}^{(p)}(x, 0)$. Then, $u$ is a continuous function in $B \backslash\{0\}$ satisfying (4.41). Finally let us prove that $u$ is a solution of the problem (1.10). Indeed, since $u$ is a positive continuous function in $B \backslash\{0\}$, then it follows from (4.41) that $V_{m, n} \psi(\cdot, u) \in L_{\text {loc }}^{1}(B \backslash\{0\})$. By $\left(\mathrm{A}_{2}\right),(3.3),\left(\mathrm{A}_{4}\right)$ and Remark 4.4, we deduce that $u \in$ $C^{2 p m-1}(\bar{B} \backslash\{0\})$. So from (4.41), we derive that for each $x \in B \backslash\{0\}$ and $0 \leq k \leq p-1$

$$
\begin{aligned}
(-\Delta)^{k m} u(x) & =b \Gamma_{m, n}^{(p-k)}(x, 0)+\int_{B} \Gamma_{m, n}^{(p-k)}(x, y) \psi(y, u(y)) d y \\
& =\Gamma_{m, n}^{(p-k)}(x, 0) L_{k} \omega_{0}(x) .
\end{aligned}
$$

Finally, using Remark 4.12 and that the set $S$ is invariant under $L_{k}$, we verify that $u$ is the required solution. This completes the proof.

Example 4.13. Assume that $n>2 m$. Let $r>n, 1 \leq \lambda<1+1 / m r$ and $\mu \geq \lambda$. Then there exists $b_{0}>0$ such that for each $b \in\left(0, b_{0}\right]$, the following nonlinear problem

$$
\begin{gathered}
(-\Delta)^{p m} u(x)=\frac{(u(x))^{\mu+1}}{(\delta(x)+u(x))^{\lambda}\left(G_{m, n}(x, 0)\right)^{\mu}}, \quad \text { in } B \backslash\{0\}, \\
\lim _{|x| \rightarrow 1} \frac{(-\Delta)^{k m} u(x)}{(1-|x|)^{m-1}}=0, \quad \text { for } 0 \leq k \leq p-1,
\end{gathered}
$$

has a positive solution $u \in C^{2 p m-1}(\bar{B} \backslash\{0\})$ satisfying for $k \in\{0,1, \ldots, p-1\}$ and all $x \in B$

$$
\frac{b}{2} \Gamma_{m, n}^{(p-k)}(x, 0) \leq(-\Delta)^{k m} u(x) \leq \frac{3 b}{2} \Gamma_{m, n}^{(p-k)}(x, 0)
$$

and for $n \geq 2(p-k) m$, we have $\lim _{|x| \rightarrow 0}\left((-\Delta)^{k m} u(x) / \Gamma_{m, n}^{(p-k)}(x, 0)\right)=b$.

Indeed, hypotheses $\left(A_{1}\right)-\left(A_{3}\right)$ are obviously satisfied and hypothesis $\left(A_{4}\right)$ follows from Corollary 2.2 and [13, lemma, page 726$]$.

\subsection{Positive solutions of the problem (1.15).}

Theorem 4.14. Assume $\left(R_{1}\right)-\left(R_{3}\right)$. Then the problem (1.15) has at least one positive solution $u \in C^{2 p m-1}(\bar{B})$, satisfying for all $k \in\{0, \ldots, p-1\}$

$$
(-\Delta)^{k m} u(x) \sim(\delta(x))^{m} .
$$


Proof. Let $K$ be a compact in $B$ such that $\gamma:=\int_{K} h(y) d y>0$ and define $r:=\min _{y \in K}$ $(\delta(y))^{m}>0$. have

By Corollary 2.2 and (3.4), there exist $c_{1}>0$ and $c_{2}>0$ such that for each $x, y \in B$ we

$$
c_{1}(\delta(x) \delta(y))^{m} \leq \Gamma_{m, n}^{(p)}(x, y) \leq c_{2} G_{m, n}(x, y)
$$

By $\left(\mathrm{R}_{2.1}\right)$ we can find $a>0$ such that $c_{1} r \gamma f(a r) \geq a$.

Since by $\left(\mathrm{R}_{2}\right)$ the function $\varphi \in K_{m, n} \subset \mathscr{F}_{m, n}^{(p)}$, then it follows by Proposition 3.3 that

$$
\beta:=\|\varphi\|_{m, n, p}<\infty
$$

By $\left(\mathrm{R}_{2.2}\right)$ we can find $b>a$ such that $c_{2} \beta g\left(2^{m} b\right) \leq b$.

Now let $\Lambda$ be the convex set given by

$$
\Lambda=\left\{u \in C(\bar{B}): a(\delta(x))^{m} \leq u(x) \leq b(\delta(x))^{m}\right\}
$$

and $T$ be the operator defined on $\Lambda$ by

$$
T u(x)=\int_{B} \Gamma_{m, n}^{(p)}(x, y) \Phi(y, u(y)) d y
$$

Using $\left(\mathrm{R}_{1}\right),\left(\mathrm{R}_{2}\right),(4.52)$ and similar arguments as in the proof of Corollary 4.3 one can verify that $T$ is a compact operator mapping $\Lambda$ into itself.

So the Schäuder fixed point theorem implies the existence of $u \in \Lambda$ such that

$$
u(x)=\int_{B} \Gamma_{m, n}^{(p)}(x, y) \Phi(y, u(y)) d y .
$$

Using $\left(\mathrm{R}_{2}\right),\left(\mathrm{R}_{3}\right)$ and similar arguments as in the proof of Theorem 4.7, it is easy to verify that $u \in C^{2 p m-1}(\bar{B})$.

Furthermore, from (1.1) we obtain that for each $k \in\{0, \ldots, p-1\}$ and $x \in B$

$$
(-\Delta)^{k m} u(x)=\int_{B} \Gamma_{m, n}^{(p-k)}(x, y) \Phi(y, u(y)) d y
$$

Finally, using (4.57), (4.52) and $\left(\mathrm{R}_{2}\right)$, we show that $u$ is the required solution.

Example 4.15. Let $r>n, \mu \in(0,1)$ and $0<\lambda<\min (\mu / 2,1 / r)$.

Then the problem

$$
\begin{gathered}
(-\Delta)^{p m} u(x)=\frac{\left(1+u^{2}(x)\right)^{\mu / 2}}{\left(\delta(x)+u^{2}(x)\right)^{\lambda}}, \quad \text { in } B \\
\lim _{|x| \rightarrow 1} \frac{(-\Delta)^{k m} u(x)}{(1-|x|)^{m-1}}=0, \quad \text { for } 0 \leq k \leq p-1,
\end{gathered}
$$


has at least one positive solution $u \in C^{2 p m-1}(\bar{B})$, satisfying

$$
(-\Delta)^{k m} u(x) \sim(\delta(x))^{m}, \quad \forall k \in\{0, \ldots, p-1\}
$$

Indeed, hypotheses of Theorem 4.14, are satisfied with $\Phi(x, t)=\left(1+t^{2}\right)^{\mu / 2} /(\delta(x)+$ $\left.t^{2}\right)^{\lambda}, \varphi(x)=1 /(\delta(x))^{\lambda+m}, g(t)=\left(1+t^{2}\right)^{\mu / 2}, f(t)=\left(1+t^{2}\right)^{\mu / 2-\lambda}$ and $h(x)$ is some positive constant.

\section{Acknowledgment}

The author is so grateful to Professor Habib Mâagli for his attention and useful discussions. He also thanks the referee for his/her careful reading of the paper.

\section{References}

[1] S. Agmon, A. Douglis, and L. Nirenberg, Estimates near the boundary for solutions of elliptic partial differential equations satisfying general boundary conditions. I, Communications on Pure and Applied Mathematics 12 (1959), 623-727.

[2] M. Aizenman and B. Simon, Brownian motion and Harnack inequality for Schrödinger operators, Communications on Pure and Applied Mathematics 35 (1982), no. 2, 209-273.

[3] I. Bachar, H. Mâagli, S. Masmoudi, and M. Zribi, Estimates for the Green function and singular solutions for polyharmonic nonlinear equation, Abstract and Applied Analysis 2003 (2003), no. $12,715-741$.

[4] T. Boggio, Sulle funzioni di Green d'ordine $m$, Rendiconti del Circolo Matematico di Palermo SerieII 20 (1905), 97-135 (Italian).

[5] H. Brezis, Analyse Fonctionnelle, Dunod, Paris, 1999.

[6] K. L. Chung and Z. X. Zhao, From Brownian Motion to Schrödinger's Equation, Grundlehren der Mathematischen Wissenschaften, vol. 312, Springer, Berlin, 1995.

[7] R. Dalmasso, Uniqueness theorems for some fourth-order elliptic equations, Proceedings of the American Mathematical Society 123 (1995), no. 4, 1177-1183.

[8] _ Existence and uniqueness of positive solutions of semilinear elliptic systems, Nonlinear Analysis. Theory, Methods \& Applications. An International Multidisciplinary Journal. Series A: Theory and Methods 39 (2000), no. 5, 559-568.

[9] D. Gilbarg and N. S. Trudinger, Elliptic Partial Differential Equations of Second Order, Grundlehren der Mathematischen Wissenschaften, vol. 224, Springer, Berlin, 1983.

[10] H.-C. Grunau and G. Sweers, Positivity for equations involving polyharmonic operators with Dirichlet boundary conditions, Mathematische Annalen 307 (1997), no. 4, 589-626.

[11] Sharp estimates for iterated Green functions, Proceedings of the Royal Society of Edinburgh. Section A. Mathematics 132 (2002), no. 1, 91-120.

[12] N. J. Kalton and I. E. Verbitsky, Nonlinear equations and weighted norm inequalities, Transactions of the American Mathematical Society 351 (1999), no. 9, 3441-3497.

[13] A. C. Lazer and P. J. McKenna, On a singular nonlinear elliptic boundary-value problem, Proceedings of the American Mathematical Society 111 (1991), no. 3, 721-730.

[14] H. Mâagli, Inequalities for the Riesz potentials, Archives of Inequalities and Applications. An International Journal for Theory and Applications 1 (2003), no. 3-4, 285-294.

[15] H. Mâagli and L. Mâatoug, Singular solutions of a nonlinear equation in bounded domains of $\mathbb{R}^{2}$, Journal of Mathematical Analysis and Applications 270 (2002), no. 1, 230-246.

[16] H. Mâagli and M. Selmi, Inequalities for the Green function of the fractional Laplacian, preprint, 2002. 
[17] H. Mâagli, F. Toumi, and M. Zribi, Existence of positive solutions for some polyharmonic nonlinear boundary-value problems, Electronic Journal of Differential Equations 2003 (2003), no. 58, 119.

[18] H. Mâagli and M. Zribi, Existence and estimates of solutions for singular nonlinear elliptic problems, Journal of Mathematical Analysis and Applications 263 (2001), no. 2, 522-542.

[19] On a new Kato class and singular solutions of a nonlinear elliptic equation in bounded domains of $\mathbb{R}^{2}$, Positivity 9 (2005), no. 4, 667-686.

[20] S. Masmoudi and M. Zribi, Positive solutions of a singular nonlinear differential operator of fourth order, Nonlinear Analysis. Theory, Methods \& Applications. An International Multidisciplinary Journal. Series A: Theory and Methods 42 (2000), no. 8, 1365-1376.

[21] L. A. Peletier and R. C. A. M. Van der Vorst, Existence and nonexistence of positive solutions of nonlinear elliptic systems and the biharmonic equation, Differential and Integral Equations. An International Journal for Theory and Applications 5 (1992), no. 4, 747-767.

[22] M. Selmi, Inequalities for Green functions in a Dini-Jordan domain in $\mathbb{R}^{2}$, Potential Analysis. An International Journal Devoted to the Interactions between Potential Theory, Probability Theory, Geometry and Functional Analysis 13 (2000), no. 1, 81-102.

[23] W. C. Troy, Symmetry properties in systems of semilinear elliptic equations, Journal of Differential Equations 42 (1981), no. 3, 400-413.

[24] N. Zeddini, Positive solutions for a singular nonlinear problem on a bounded domain in $\mathbb{R}^{2}$, Potential Analysis. An International Journal Devoted to the Interactions between Potential Theory, Probability Theory, Geometry and Functional Analysis 18 (2003), no. 2, 97-118.

[25] Q. S. Zhang and Z. X. Zhao, Singular solutions of semilinear elliptic and parabolic equations, Mathematische Annalen 310 (1998), no. 4, 777-794.

[26] Z. X. Zhao, Green function for Schrödinger operator and conditioned Feynman-Kac gauge, Journal of Mathematical Analysis and Applications 116 (1986), no. 2, 309-334.

Imed Bachar: Département de Mathématiques, Faculté des Sciences de Tunis, Campus Universitaire, 2092 Tunis, Tunisia

E-mail address: imed.bachar@ipeit.rnu.tn 


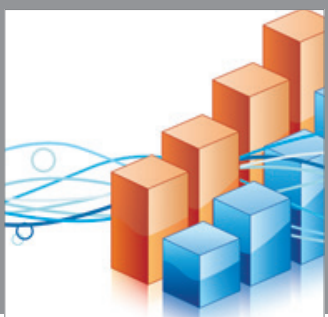

Advances in

Operations Research



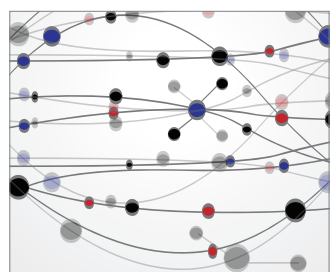

\section{The Scientific} World Journal


International Journal of

Mathematics and

Mathematical

Sciences
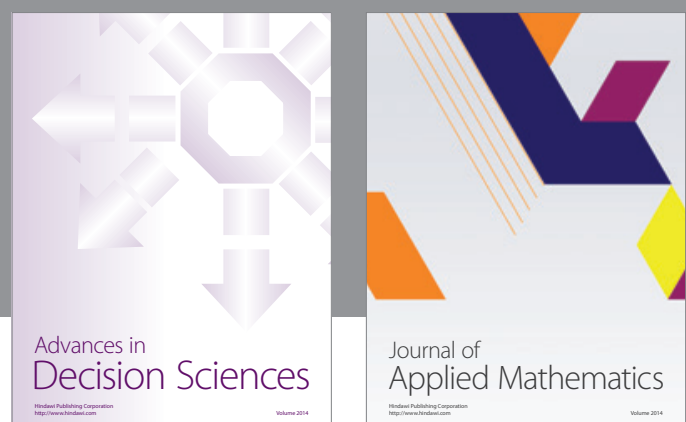

Journal of

Applied Mathematics
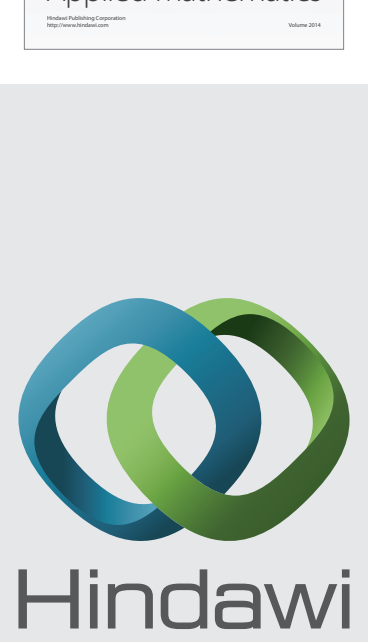

Submit your manuscripts at http://www.hindawi.com
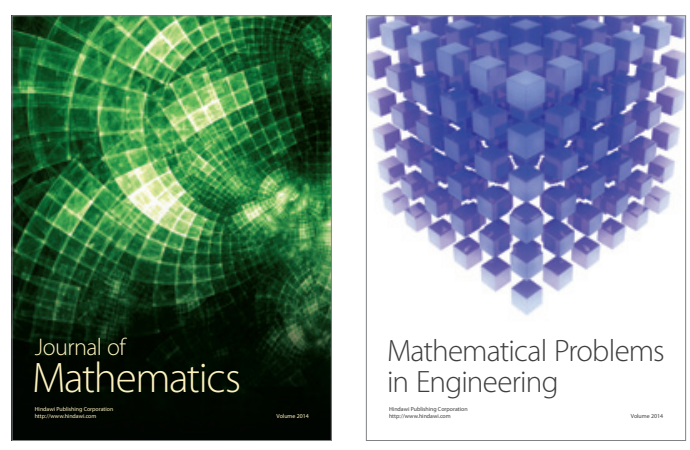

Mathematical Problems in Engineering
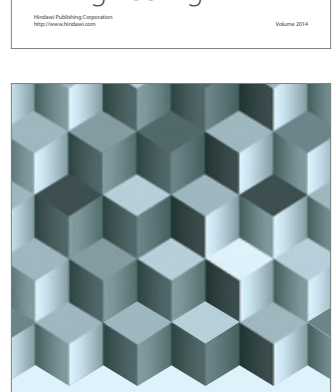

Journal of

Function Spaces
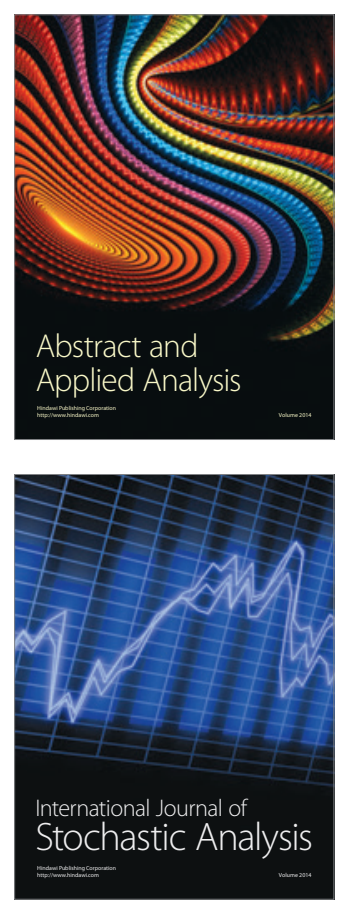

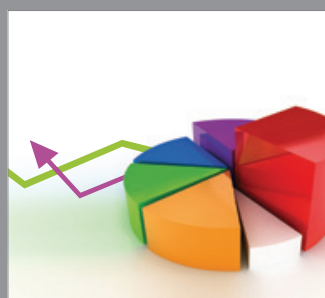

ournal of

Probability and Statistics

Promensencen
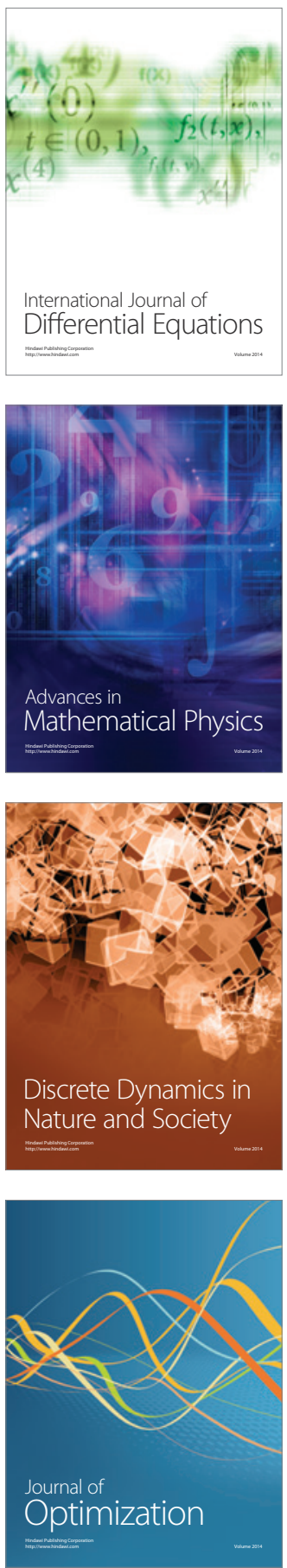